\title{
A Semantic Meta-data Describing Video Information Based on Ontology
}

\author{
Wang Yang ${ }^{1}$, Song Wen-fei ${ }^{2,}$ and Liu Yan-ju ${ }^{3 *}$ \\ ${ }^{1}$ School of economics and management, Qiqihar University, Qiqihar, China \\ ${ }^{2}$ Zhejiang Industry Polytechnic College, Shaoxing, China \\ ${ }^{3}$ Computer Center, Qiqihar University, Qiqihar, China \\ *15146692464@163.com
}

\begin{abstract}
The paper has investigated the meta-data for annotating the video information, and put up with the framework architecture of a semantic meta-data describing video information based on ontology. Firstly a domain ontology library is constructed, the video information is described by the ontology, and the video library system with architecture is developed. The paper builds a semantic ontology library for Beijing Opera, and gets the conclusion that it is feasible to describing video information with semantic meta-data based on ontology.
\end{abstract}

Keywords: Semantic Meta-data, ontology, video information

\section{Introduction}

Meta-data is a kind of data which can be used to describe and locate resources. Meta-data has functions of retrieving, using, and managing information unit. At present, the type of meta-data is included mainly as following:

a. Descriptive meta-data. It is used for content description and identification information resources, properties and characteristics of meta-data.

b. Administrative meta-data. It is used to manage information resources meta-data.

c. Applied Meta-data, such as saving and using meta-data information resources.

Terms of meta-data standards has been researched and developed in the whole world. Take Dublin Core meta-data Element Set (DC for short) for example, it is the most widely used in research and application in the field of library study sessions in the library [1]. DC meta-data consists of fifteen content elements, and describes resources from aspect of title, creator and other 13 elements, and these 15 core meta-data elements are the basement of research on video information description [2]. Another example is the MPEG-7 (Multimedia Content Description Interface), which is regulated by Moving Picture Experts Group (MPEG) as a description of the development of multi-media data, and it's purpose is to provide a standardized core technology, further to make audio data content in visual multimedia environment to get standardized description[3], Open Video Project set by American College of Information and Library University of North Carolina, video meta-data scheme of which is based on MPEG-7 to achieve digital video sharing [4].

Among domestic researches on meta-data, the largest scale project is China Digital Library Standards Construction Project which started in October of 2002, and this project developed a series of digital resources and processing practices, basic meta-data, specialized meta-data specification [5], etc. All of these have been widely used in domestic digital library community. While in the field of multimedia cataloging rules, some description specifications and rules of digital information have been developed, like 
a meta-data map, audio and other forms, but standards for video information have not been.

In systems whose information is organized by meta-data, information retrieval use query strategy of entity matching in projects of meta-data marking, and it lacks of inter-related information on the semantic level. And for video information, we believe it's necessary to give Meaning Expression to scene, the story units, and even the lens or frame. Based on the above, this paper proposed that using semantic meta-data to describe the video information strengthen inter-related information by ontology, and further to form a video system with construction.

\section{Ontology}

Ontology is a philosophical concept, It was Neches that earliest introduce ontology technology into artificial intelligence in 1991 [6]. And then, ontology started to be active in researching fields of Artificial intelligence, knowledge engineering, information science and etc. In 1998, Studer set up the definition of ontology which is accepted widely nowadays, and it is consisted four meanings: concept ululation, Explicit, Formal and Share [7]. The conception of ontology was abstracted from the objective world, it's clear, and sharing, and can be digitized and processed by computers through the knowledge system developed by ontology.

\subsection{Consists of Ontology}

Perez [8] et al. proposed to organize the conception of ontology by the method of classification, consists Concept, Relations, Function and Instances, and they can be expressed by five-tupelos Model ----- $\{\mathrm{C}, \mathrm{R}, \mathrm{F}$, Ao, In $\}$.

(1) $\mathrm{C}$ represents a set of concepts, which include a wide range of things, like representing the behavior, the function, and the reasoning process of the above.

(2) R represents a set of relationships, which includes relationships among concepts in the same knowledge field, and from the aspect of collection, it's a collection of relations, like affiliation relationship, inheritance relationship and etc.

(3) F represents function, which is one special one in relationship, it's definition is that: the $\mathrm{n}^{\text {th }}$ element is determined by the $(\mathrm{n}-1)^{\text {th }}$ element.

(4) Ao represents ontology axioms, that means the rules of definition are always been set up in conception and property.

(5) In represents Instances, which is the concrete objective of the definition.

The building of ontology has property of field, which is an effective tool on conceptual and knowledgeable level, it aims at defining knowledge of connective fields, offer the common understanding of the knowledge field, and determine conceptions accepted widely in the field, further to describe definitions and relationship among them, to realize the knowledge sharing in the knowledge field.

\subsection{Description Language of Ontology}

Description language of ontology is rule which used to build the ontology, which is the basement of it. The description of ontology can be realized by natural languages, and relevant description languages as well. With researches of Scientists, there generated lots of ontology description languages, like KIF, Onto-lingua and etc. With the development of Web technology, generated description language of ontology based on Web, like RDF, RDFS, OWL and etc., which can realize information sharing and exchanging better. Figure 1 is a diagram of various types of ontology description languages.

The following examples would analyses several essential ontology languages: 


\section{(1) XML}

XML(Extensible Markup Language) [9] is one marking implementation language, which is used to mark electronic documents with structure, is one source language can be customized my users, and it can support grammars of other languages. XML would provide a unified approach to describe and exchange structured data with application-independence.

\section{(2) OWL}

OWL (Web Ontology Language) is one standard ontology language, which is used to publish and share on Web. It was extended and developed on the basis of RDF, and more powerful in semantic description than RDF and XML. And in application, OWL has three sub-languages with different expression [10].

OWL Lite, is the simplest kind of sub-language in syntax. It provides primarily ontology users who needs simply inheritance or constraints. OWL DL is on the basis of Description Logics, with the most powerful expressing capability. OWL Full can be used to finish the ontology building with the most powerful expressing capability, whose grammar is absolutely free in expressing, suitable for high expressing occasions.

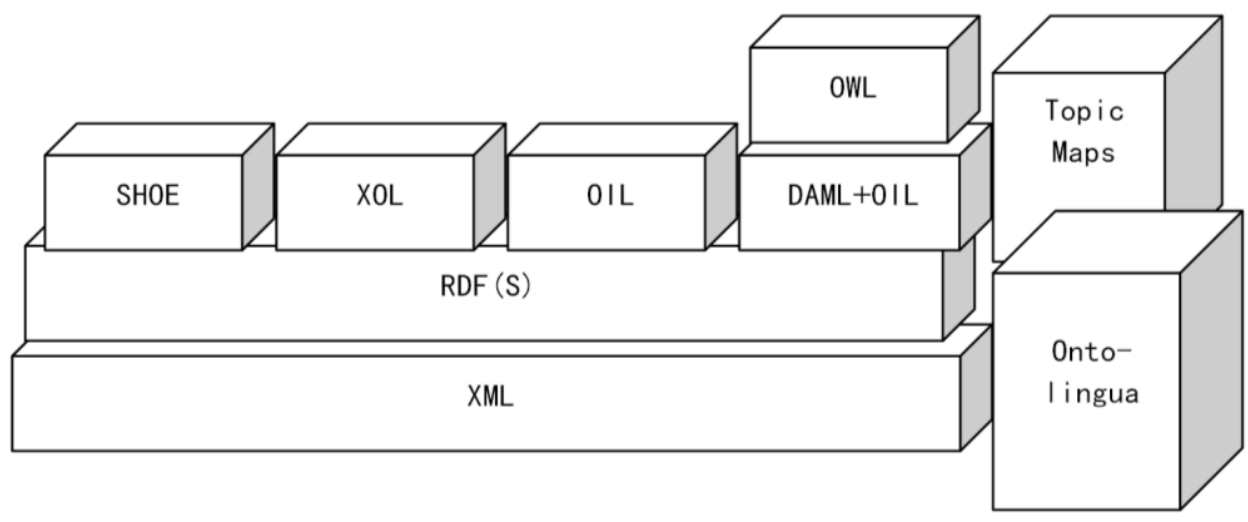

Figure 1. Diagram of Various Types of Ontology Description Languages

\section{(3) $\mathrm{RDF}$}

RDF (Resource Description Framework) is describing framework of resources, which is one standard developed on the basis of XML by W3C since 1999, which is used to compensate for the limitations of XML.

a. RDF is one Semantic Meta-data model for expressing the objectives of web resources and their relationships.

b. Attribute, which defines the various aspects of resources and entities, including features, attributes and relationships.

c. Statement, which consists of themes, predicates and objects, its destinations attribute values to resources and entities.

\section{Description of Semantic Meta-data}

\subsection{Semantic Meta-data based on Ontology}

The description of video semantic information not only needs description of its content, but more requires a combination of retrieval, storage, tables, its sources and relevant information. Describing the information need more semantic and machine-understandable, while the traditional text-based meta-data do not have these 
features. Thus we need to introduce ontology into semantic meta-data, and use languages with more powerful expressing capability to express semantic meta-data, and furthermore, the structured hierarchy and seasoning capabilities of these languages can make the meta-data using more flexible, which is the new request of meta-data using in information describing under semantic web environment [11-12].

We call the semantic meta-data as SMD. Semantic meta-data can redefine the basis meta-data, and they can realize sharing and universal in heterogeneous environment, and can inter-operate at the semantic level. This realize meta-data's extension in grammar, structure and semantic, and under the heterogeneous environment, it can give more accurate, effective description, management and using, further to realize flexible exchange [13-14].

\subsection{Description Framework of Semantic Meta-data}

We believe it's necessary to give a further semantic description to the scenes, story units, lens and even frames. Based on this, this paper represents that using Semantic Meta-data to describe video information in core meta-data of MPEG-7, and through ontology to strengthen inter-related information, further to form video base with system structure. The following is description framework of Semantic meta-data.

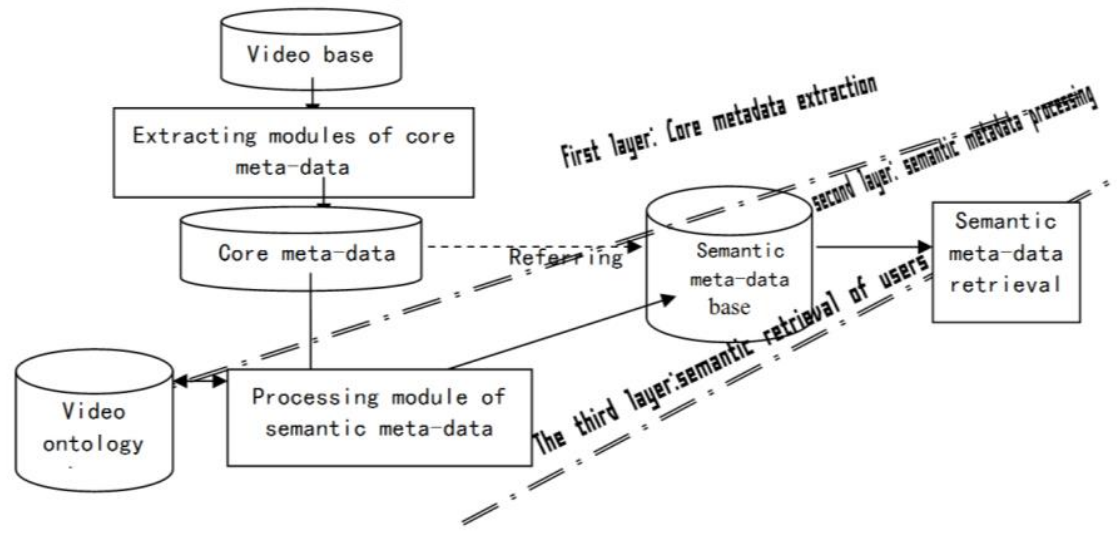

Figure 2. Description Framework of Semantic Meta-data based on Ontology

There are there layers in the whole framework.

The first layer would realize extraction of core meta-data. In this layer, we would analyze core meta-data of video MPEG-7 in video base, and finish the collection of video's core meta-data。

The second layer would realize the semantic meta-data description of video information. Through the video information base built before head, the processing module of semantic meta-data would compare core meta-data and definitions in the base, and the core meta-data keywords are mapped to the concept of semantic meta-data, then form into semantic concept hierarchy.

The third layer is for users' retrieval that is retrieving video information from Semantic Meta-data with system structure. In this system of framework, we can realize recommended business initiatively by operation and interests on relevant video information.

\subsection{Domain Video Ontology Base}

Beijing Opera is a representative Chinese opera, also loved by many international friends. In this paper, take the Beijing Opera video as an example to construct ontology, 
start from the video content information, and from the story, characters, contents, time, venue, schools etc. to build ontology base for video description [14].

\section{(1) Characters ontology base}

Various characters' participating in video, including directors, screenwriters, role, musicians etc., structure system is as follows:

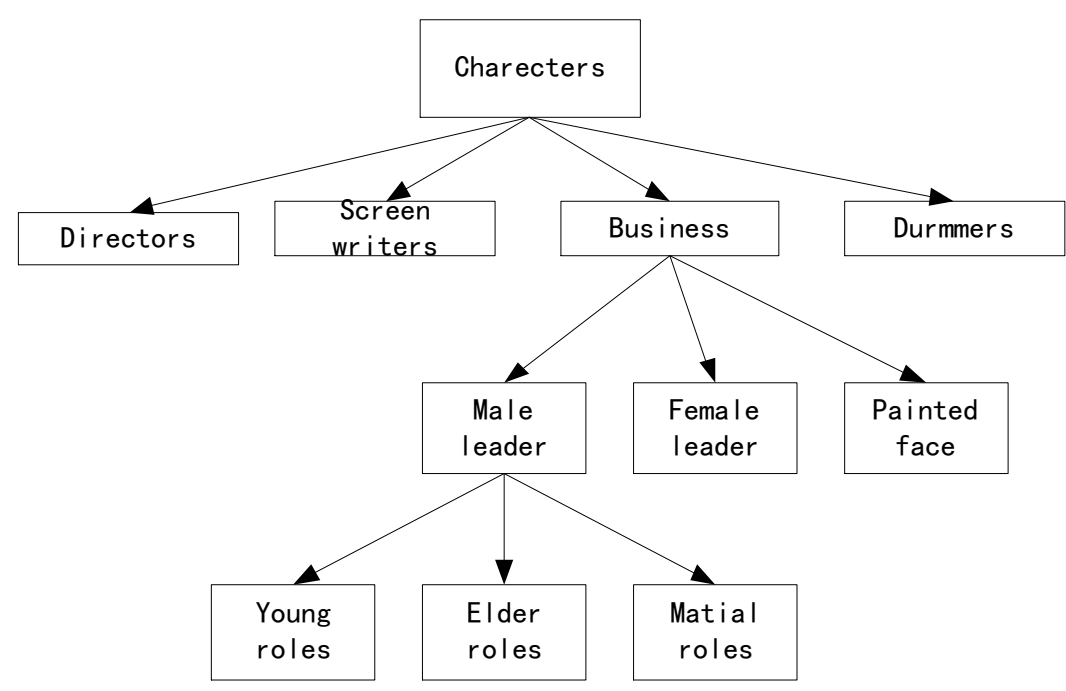

Figure. 3 Characters Ontology Base

\section{(2) Genres Ontology Base}

At present, there are many prominent artists who made a great contribution, based on their artistic style, to form their own genres, such as Yangxiao Lou's Yang School, Mei Lanfang's Mei School.

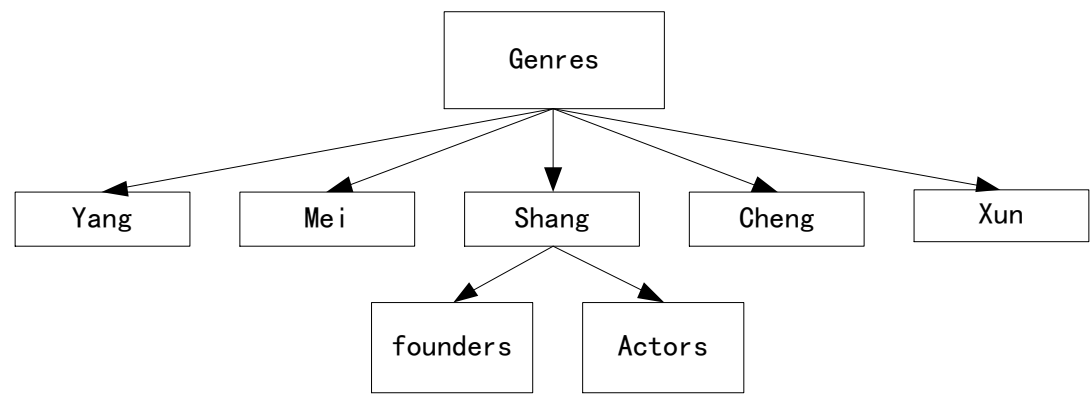

Figure 4. Genres Ontology Base

(3) Performance form ontology base

The performance form of Beijing opera includes Chang, Nian, Zuo, Da, and Wu. Chang is singing, Nian is speaking, Zuo is performing actions, Da is fighting, and Wu is dancing. 


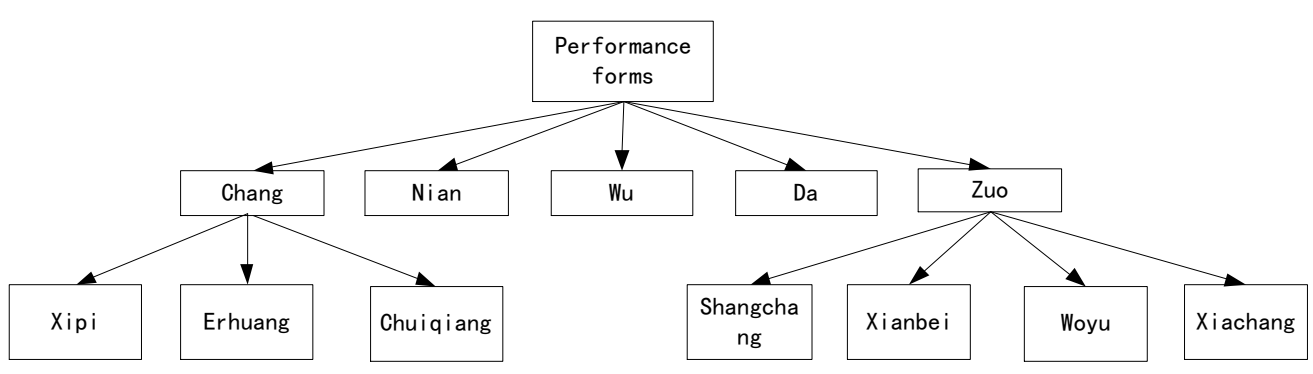

Figure 5. Performance Form Ontology Base

\subsection{Semantic Metadata Description of Video}

The first thing to extract semantic metadata of video information is to segment the video. Take the "Drunken Beauty" as an example, The "Drunken Beauty" is the representative work of the Mei school, the video is part of the one. This video is the famous MingJiaMingDuan CCTV program, Liang Weiling, Zhang Bin, Zhang Bing of Hebei Beijing opera play the roles of Yang Yuhuan (Huandan), Gao Lishi (Wenchou), Pei Lishi (Wenchou) respectively, includes the performing forms of Nian, Zuo and Wu, such as the famous Xianbei, Woyu etc. According to performing form of Beijing opera, the video is split and described by using the semantic metadata ontology base, see Table 1 .

Table 1. Semantic Meta-data Description of Video

\begin{tabular}{|c|c|c|l|}
\hline Time $(\mathrm{m}: \mathrm{s})$ & Characters & $\begin{array}{c}\text { Performing } \\
\text { form }\end{array}$ & \multicolumn{1}{|c|}{ Performing contents } \\
\hline $0: 00$ & Yang Yuhuan & solo dance & \\
\hline $1: 35-1: 45$ & Yang Yuhuan & Zuo & Wo yu \\
\hline $2: 43-3: 01$ & Yang Yuhuan & Zuo & Wo yu, Smell flowers \\
\hline $3: 22$ & Pei Lishi & Zuo & Appear on the stage \\
\hline $3: 25-3: 27$ & Pei Lishi & Nianbai & Gaolishi invites the queen to drink. \\
\hline $3: 37-3: 39$ & Pei Lishi & Nianbai & Queen, wine is here, please drink it. \\
\hline $3: 54-4: 00$ & Pei Lishi & Nianbai & $\begin{array}{l}\text { Oh, wine burst. Queen, wine is } \\
\text { here, please drink it. }\end{array}$ \\
\hline $4: 25-4: 45$ & Yang Yuhuan & Zuo & Cup in mouth. \\
\hline $4: 47$ & Pei Lishi & Zuo & Go off stage \\
\hline $4: 54$ & Gao Lishi & Zuo & Appear on the stage \\
\hline $4: 54-5: 00$ & Gao Lishi & Nianbai & Gaolishi invites the queen to drink. \\
\hline $5: 10-5: 13$ & Gao Lishi & Nianbai & Queen, wine is here, please drink it. \\
\hline $5: 22-5: 30$ & Gao Lishi & Nianbai & $\begin{array}{l}\text { Oh, wine burst. Queen, wine is } \\
\text { here, please drink it. }\end{array}$ \\
\hline $5: 44-6: 04$ & Yang Yuhuan & Zuo & Cup in the mouth \\
\hline $6: 05$ & Gao Lishi & Zuo & Go off stage \\
\hline $6: 13$ & court ladies & Zuo & Appear on the stage \\
\hline $6: 14-6: 17$ & court ladies & Nianbai & Court maids toast \\
\hline $6: 23-6: 26$ & court ladies & Nianbai & Queen, wine is here, please drink it. \\
\hline $6: 33-6: 36$ & court ladies & Nianbai & Queen, wine is here, please drink it. \\
\hline $6: 55-7: 23$ & Yang Yuhuan & Zuo & Cup in the mouth \\
\hline & \multicolumn{2}{|r}{} \\
\hline
\end{tabular}

If users want to retrieve the relevant video of Yang Yuhuan, Xianbei, through semantic meta-data's guidelines, it will give three video clips 4:25-4:45, 5:44-6:04, 6:55-7:23 in the video; for video library, it will retrieve the performing fragments of different theaters, different artists, and is helpful for users, especially for professional performers further appreciating and studying them. 


\section{Conclusion}

Video information has territoriality, and there are different ontology bases for different video information. To describe the video's content more accurately and more friendly, we need territoriality experts to join the building of semantic ontology base which would offer one division in description of video information. This paper presents that using semantic meta-data to describe video information, split video clips and describe the semantic meta-data by building ontology base for Beijing opera video, then form the video base with system structure and can be personalized service to uses.

\section{Acknowledgements}

This work was jointly supported by the National Natural Science Foundation of China (Grant No.61403222), Natural Science Foundation of Heilongjiang Province (Grant No. F201439, F201440).

\section{References}

[1] ISO-15836-2009, Information and documentation -- The Dublin Core metadata element set.

[2] Z. Liu, "Design and Development for Video Files Meta-data [D], Shandong University, (2009).

[3] J. Zhang, M. Duan and Z. Shen, "Project Summary of Foreign Video and Audio Meta-data Researches [J]", Library Tribune, vol. 6, no. 25, (2005).

[4] The Open Video Project: A shared digital video collection, http://www.open-video.org/, (2012).

[5] China Digital Library Standards Development, http://cdls.nstl.gov.cn, (2014).

[6] S. William and T. Austin, "Ontologies [J]”, IEEE Intelligent Systems, vol. 1, no. 2, (1999).

[7] R. Studer, VR. Benjatnins and D. Fensel, "Knowledge Engineering: principles and methods [J]", Data and Knowledge Engineering, vol. 122, no. 25, (1998).

[8] A. G. Perez and V R. Benjamins, "Overview of Knowledge Sharing and Reuse Component: overview [EBOL]", http://www.w3.org/TR/2003/WD-owl-features-20030331, (2013).

[9] L. Wang, "Research and Application on Semantic Retrieval for Digital Library Based on Ontology [D]", Zhengzhou: Zhengzhou University, (2011).

[10] D. L. McGuinness and F. V. Harmelen, "OWL web ontology language Ontologies and Problem-solving Methods [C]", Workshop on Ontologies Problem-Solving Methods: Lessons Learned and Future Trends (IJCAI99), (1999), October, 32-40, Agosto, Estocolmo.

[11] F. Tao, N R. Shadbolt and L. Chen, "Semantic web based content enrichment and knowledge reuse in e-science [C]", Proceedings of 3rd International Conference on Ontologies, (2004), September 12, Larnaca, Cyp-rus.

[12] V. Parekh, J.-P. Gwo and T. Finin, "Ontology based semantic metadata for geoscience data [C]", Proceedings of International Conference of Information and Knowledge Engineering Date, Las Vegas Nevada, USA, (2004) July 14, pp. 308-312.

[13] K. Sivashanmugam, A. Sheth and J. Miller, "Metadata and semantics for web services and processes [EB/OL]", http://lsdis.cs.uga.edu/lib/download/Schlageter-book-chapter-final.pdf

[14] A. Sheth, "Semantic metadata for enterprise information integration [EB/OL]", http://www. dmreview.com/article_sub.cfm? ar-ticleId=6962, (2014). 
International Journal of Multimedia and Ubiquitous Engineering Vol.10, No.5 (2015) 